\title{
Dengue vascular leak syndrome: insights into potentially new treatment modalities
}

\author{
Anna P. Durbin \\ Bloomberg School of Public Health, Johns Hopkins University, Baltimore, Maryland, USA.
}

\begin{abstract}
Dengue viruses (DENV) are the most common cause of mosquito-borne viral illness in the world, affecting approximately $\mathbf{4 0 0}$ million people annually. Symptomatic illness ranges from a mild, self-limiting febrile illness to one manifested by plasma leakage that can lead to vascular collapse and death. In this issue of the $J C l$, Rathore et al. report that DENV can cause mast cell degranulation independently of mast cell infection, resulting in the release of the vasoactive mediators chymase and tryptase. The authors showed that recombinant chymase and tryptase increased endothelial permeability in a dose-dependent manner in human microvascular endothelial cells. They went on to evaluate the tryptase inhibitor nafamostat mesylate in a mouse model for severe DENV viremia. Strikingly, the potential therapeutic prevented and reversed the tryptase-induced vascular permeability. As there are currently no licensed drugs for the treatment of dengue, these findings present a possible treatment modality for severe disease.
\end{abstract}

\section{Mechanism for vascular leakage}

Nearly two-thirds of the world's population are living in dengue-endemic regions and are at risk of acquiring the disease (1). Twenty-five percent of the estimated 400 million people who annually develop dengue infections present for clinical care (2). Common manifestations of severe dengue include thrombocytopenia and hemorrhagic symptoms and death. Epidemiologic studies suggest that severe dengue most commonly results from a secondary heterotypic dengue infection (3). It is thought that, during the primary dengue infection, dengue virus (DENV) binds a nonneutralizing heterotypic antibody. Next, the antibody-virus complex binds the Fc $\gamma$ receptor and enters cells, a phenomenon known as antibody-dependent enhancement of infection $(4,5)$. Theoretically, enhanced infection of target cells results in greater viral replication and pathogenicity, with higher virus titers associated with more severe disease (6).

The process by which DENV causes endothelial cell dysfunction and vascular leak has long been investigated. In this issue, Rathore and colleagues identify a mechanism by which DENV promotes vascular pathology and shock (7). In severe dengue disease, plasma leakage occurs later in infection, after the waning of fever and viremia. As such, plasma leakage is likely due to host-immune or mediatorinduced response as opposed to endothelial cell infection and consequent destruction (8). In contrast to other hemorrhagic fever viruses, such as Ebola, in which endothelial cells remain intact, Rathore et al. suggest that DENV causes separation of endothelial cell junctions, which results in plasma leakage (7).

It was long hypothesized that cytokine storm induced by crossreactive $\mathrm{T}$ cells stimulated during a second, hetero-

Related Article: p. 4180

Conflict of interest: The author has declared that no conflict of interest exists.

Copyright: @ 2019, American Society for Clinical Investigation.

Reference information: J Clin Invest. 2019;129(10):4072-4073. https://doi.org/10.1172/JCI131170.

typic dengue infection mediates vascular leak syndrome. However, such a dramatic hemorrhagic manifestation can also occur in primary dengue infection in which crossreactive T cells are not present (9). In addition, treatment trials using corticosteroids to combat cytokine storm, thereby preventing or treating dengue-associated vascular leak syndrome, failed (10).

Rathore and colleagues reported high levels of chymase and tryptase in the blood of patients following dengue infection. Previously, high levels of both, but more specifically tryptase, have been correlated with more severe disease $(11,12)$. Mechanistically, the study authors hypothesize that tryptase induces increased vascular permeability and leakage by reducing the expression of the cellular adhesion molecule CD31 on endothelial cells, resulting in the loss of tight junctions. Importantly, the authors demonstrated the effect of tryptase in both in vitro and in vivo models (7).

\section{Potential DENV treatment modalities}

Currently, the only treatment for dengue vascular leak syndrome is supportive care. Although a vaccine for the prevention of dengue has been licensed, it is only recommended for those who have previously had a dengue infection. While other vaccines are in clinical trials, unprevented cases of dengue still require treatment. For this reason, medications that can treat dengue and/or vascular leak complications are needed.

Recent evidence has also implicated the non-structural protein 1 (NS1) and mast cells as factors involved in causing endothelial dysfunction. NS1 mediates vascular leakage either through TLRs $(13,14)$ or by inducing the expression of human sialidases, resulting in the degradation of the endothelial glycocalyx and leading to vascular leak. Glasner et al. demonstrated that mice that received a cocktail comprising the sialidase inhibitor, a cathepsin inhibitor, and hepara- 
nase were protected from vascular leak induced by dengue (14). Rathore et al. show that there are likely other biologic contributors to the vascular leak syndrome in dengue. Notably, in the DENV mouse model, treatment with nafamostat mesylate for 24 to 48 hours reversed endothelial cell junction separation and resolved plasma leakage (7).

The work of Rathore et al. lays the groundwork for further evaluation of tryptase inhibition for limiting the adverse effects of severe dengue. One of the next steps toward finding a safe and effective drug for treating dengue-induced vascular leak syndrome is to evaluate nafamostat mesylate in human clinical trials. It is critical that these findings be translated into clinical action.

Address correspondence to: Anna P. Durbin, 624 North Broadway, Room 251, Baltimore MD 21205. Phone: 410.614.4736; Email: adurbin1@jhu.edu.
1. Brady OJ, et al. Refining the global spatial limits of dengue virus transmission by evidence-based consensus. PLoS Negl Trop Dis. 2012;6(8):e1760.

2. Bhatt $S$, et al. The global distribution and burden of dengue. Nature. 2013;496(7446):504-507.

3. Burke DS, Nisalak A, Johnson DE, Scott RM. A prospective study of dengue infections in Bangkok. Am J Trop Med Hyg. 1988;38(1):172-180.

4. Halstead SB, Nimmannitya S, Cohen SN. Observations related to pathogenesis of dengue hemorrhagic fever. IV. Relation of disease severity to antibody response and virus recovered. Yale J Biol Med. 1970;42(5):311-328.

5. Halstead SB. Observations related to pathogensis of dengue hemorrhagic fever. VI. Hypotheses and discussion. Yale J Biol Med. 1970;42(5):350-362.

6. Vaughn DW, et al. Dengue viremia titer, antibody response pattern, and virus serotype correlate with disease severity. JInfect Dis. 2000;181(1):2-9.

7. Rathore APS, et al. Dengue virus-elicited tryptase induces endothelial permeability and shock. J Clin Invest. 2019;129(10):4180-4193.

8. Green S, et al. Early immune activation in acute dengue illness is related to development of plasma leakage and disease severity. JInfect Dis. 1999;179(4):755-762.
9. Halstead SB. Pathogenesis of dengue: challenges to molecular biology. Science. 1988;239(4839):476-481.

10. Tam DT, et al. Effects of short-course oral corticosteroid therapy in early dengue infection in Vietnamese patients: a randomized, placebo-controlled trial. Clin Infect Dis. 2012;55(9):1216-1224.

11. St John AL, Rathore AP, Raghavan B, Ng ML, Abraham SN. Contributions of mast cells and vasoactive products, leukotrienes and chymase, to dengue virus-induced vascular leakage. Elife. 2013;2:e00481.

12. Tissera H, et al. Chymase level is a predictive biomarker of dengue hemorrhagic fever in pediatric and adult patients. J Infect Dis. 2017;216(9):1112-1121.

13. Beatty PR, Puerta-Guardo H, Killingbeck SS, Glasner DR, Hopkins K, Harris E. Dengue virus NS1 triggers endothelial permeability and vascular leak that is prevented by NS1 vaccination. Sci Transl Med. 2015;7(304):304ra141.

14. Glasner DR, Ratnasiri K, Puerta-Guardo H, Espinosa DA, Beatty PR, Harris E. Dengue virus NS1 cytokine-independent vascular leak is dependent on endothelial glycocalyx components. PLoS Pathog. 2017;13(11):e1006673. 